\title{
Instabilities in a dissipative soliton-similariton laser using a scalar iterative map
}

\author{
Fanchao MenG ${ }^{1}$, Coraline Lapre ${ }^{1}$, Cyril Billet ${ }^{1}$, GoËry Genty ${ }^{2}$, And John M. Dudley ${ }^{1}$ \\ ${ }^{1}$ Institut FEMTO-ST, Université Bourgogne Franche-Comté CNRS UMR 6174, Besançon, 25000, France \\ ${ }^{2}$ Photonics Laboratory, Tampere University, Tampere, Fl-33104, Finland \\ *Corresponding author: john.dudley@univ-fcomte.fr
}

\begin{abstract}
Numerical simulations of a dissipative solitonsimilariton laser are shown to reproduce a range of instabilities seen in recent experiments. The model uses a scalar nonlinear Schrödinger equation map, and regions of stability and instability are readily identified as a function of gain and saturable absorber parameters. Studying evolution over multiple roundtrips reveals spectral instabilities linked with soliton molecule internal motion, soliton explosions, chaos, and intermittence. For the case of soliton molecules, the relative phase variation in the spectrum is shown to be due to differences in nonlinear phase evolution between the molecule components over multiple roundtrips.
\end{abstract}

There is currently much interest in the real-time characterization of mode-locked dissipative soliton fiber laser dynamics. Dispersive Fourier Transform measurements of spectral variations are a particular focus, revealing soliton build up dynamics [1-5], soliton molecules with internal motion [6, 7], and soliton explosions and pulsations [8-14]. Work has also reported complex intermittence where a laser randomly alternates between sequences of metastable molecules, explosions, and chaos [15].

Although measuring such instabilities has been relatively straightforward, interpreting the underlying physics has proven more difficult. Certainly insight has been obtained from complex cubic quintic Ginzburg-Landau equation (CGLE) studies [16-19], as well as iterative map simulations based on nonlinear Schrödinger equation (NLSE) modelling for fiber segments, and suitable transfer functions for other elements [6, 8, 20-22]. However, although these approaches have reproduced some results seen experimentally, simulations have generally considered specific descriptions for key components, such as saturable absorption based on nonlinear loop mirrors [22], vector polarization evolution [20], or rate-equation gain dynamics [6, 8]. The question remains to what degree instabilities observed experimentally can be reproduced using more generic models.

In this Letter, we simulate a dissipative soliton laser using an iterative cavity map based on only scalar NLSE propagation and widely-used models for gain and saturable absorption.

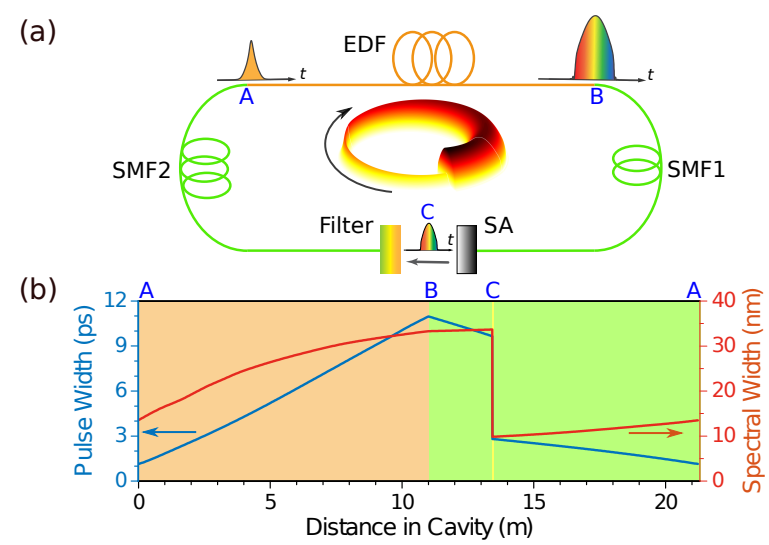

Fig. 1. (a) Schematic: EDF, erbium doped fiber; SMF, single mode fiber; $\mathrm{SA}$, saturable absorber. $L_{\mathrm{AB}}=11 \mathrm{~m}$ with $\beta_{2}=$ $+40 \times 10^{-3} \mathrm{ps}^{2} \mathrm{~m}^{-1}, \gamma=6 \times 10^{-3} \mathrm{~W}^{-1} \mathrm{~m}^{-1}$. L $\mathrm{SMF}_{\mathrm{S} 1}=2.42 \mathrm{~m}$ and $L_{\mathrm{SMF} 2}=7.8 \mathrm{~m}$ with $\beta_{2}=-21.7 \times 10^{-3} \mathrm{ps}^{2} \mathrm{~m}^{-1}$ and $\gamma=1.1 \times 10^{-3} \mathrm{~W}^{-1} \mathrm{~m}^{-1}$. Net cavity GVD is $+0.22 \mathrm{ps}^{2}$. (b) Evolution of temporal (blue, left axis) and spectral (red, right axis) widths for stable single pulses $\left(q_{0}=0.9, P_{\text {sat }}=150 \mathrm{~W}, E_{\text {sat }}=\right.$ $0.4 \mathrm{~nJ}, g_{0}=0.78 \mathrm{~m}^{-1}$ ).

Although saturable absorbers in many lasers use nonlinear polarization evolution, our ability to reproduce instabilities seen in experiments with a scalar model highlights the role of basic NLSE dynamics. Moreover, we generate a detailed stability map of laser operation, and explicitly reproduce both internal motion dynamics of soliton molecules, and complex sequential instabilities.

We consider a soliton-similariton laser [23, 24] as in Fig. 1, with normal group velocity dispersion (GVD) Erbium-doped fiber (EDF), anomalous GVD single-mode fibre (SMF1 and SMF2), a saturable absorber (SA), and a spectral filter. The parameters (see caption) are adapted from Ref. [15]. Each fiber segment is modelled by a modified scalar NLSE [23]:

$$
\frac{\partial A}{\partial z}=-\frac{i \beta_{2}}{2} \frac{\partial^{2} A}{\partial \tau^{2}}+\frac{\hat{g}}{2} A+i \gamma|A|^{2} A
$$

Here, $A=A(z, \tau)$ is the pulse envelope with $z$ the propagation coordinate and $\tau$ co-moving time. $\beta_{2}$ and $\gamma$ are the GVD and nonlinearity parameters for each segment. To study the 
ability of the simplest model to reproduce complex dynamics, higher-order terms are not included. The EDF gain is: $\hat{g}(\omega)=g_{0} /\left(1+E / E_{\text {sat }}\right) \times 1 /\left(1+\Omega^{2} / \Omega_{\mathrm{g}}^{2}\right)$ where $g_{0}$ is the unsaturated (small signal) gain, and $\Omega_{\mathrm{g}}$ corresponds to a $40 \mathrm{~nm}$ gain bandwidth. $E=\int|A|^{2} d \tau$ is the intracavity energy, and $E_{\text {sat }}$ is a gain saturation parameter [25]. $\Omega=\omega-\omega_{0}$ is the (angular) frequency detuning, with $\omega_{0}$ corresponding to a wavelength of $1550 \mathrm{~nm}$. The saturable absorber is described by the function [23]: $T(\tau)=1-q_{0}\left(1+P(\tau) / P_{\text {sat }}\right)^{-1}$ where $q_{0}$ is modulation depth, $P(\tau)=|A(z, \tau)|^{2}$ is instantaneous power, and $P_{\text {sat }}$ is saturation power. We use $q_{0} \geq 0.7$, consistent with previous modelling of similar lasers [26]. The spectral filter was a $10 \mathrm{~nm}$ intensity FWHM supergaussian with unity transmittance at line center. A lumped linear intracavity loss of $2.2 \mathrm{~dB}$ was applied after the filter to model output coupling and insertion losses. Simulations used $2^{13}$ points, a 500 ps time span, and adaptive spatial steps.

Figure 1(b) plots stable single pulse intracavity evolution. In this regime, this model has previously reproduced linearlychirped parabolic pulses as seen in experiment [15]. To study instabilities, we systematically varied the saturable absorber and gain parameters to identify regimes with qualitatively different behaviour. Figure 2 shows the different stability regions identified (see legend and caption) as a function of: (a) saturable absorber parameters $\left(q_{0}, P_{\text {sat }}\right)$ at a fixed gain setting and (b) gain parameters $\left(g_{0}, E_{\text {sat }}\right)$ for a fixed saturable absorber setting. The parameter range considered encompassed values used in similar studies $[21,23,26]$.

The initial conditions used for Fig. 2 were single and double gaussian pulses (2 ps FWHM), injected at Point A in Fig. 1. The parameter spaces were finely gridded and the behaviour of the model was studied towards either convergence or instability. For some parameters, the seed rapidly decayed, corresponding to the below-threshold region. We sought convergence up to $10^{5}$ roundtrips but in many cases, steady state was reached at only several 100's of roundtrips. We describe Fig. 2 as follows. The green region corresponds to convergence towards a stable single pulse. These single pulse states were found to be insensitive to a wide range of initial conditions: pulse durations of 1-30 ps and peak powers of $0.1 \mathrm{~mW}-10 \mathrm{~W}$. Even when seeded from noise, the simulations still converged to a final state depending only on the laser parameters. Noise seeds used ranged from a one photon per mode background to $100 \%$ (uniformly distributed) random intensity modulation.

The orange region corresponded to either stable single pulses or molecules depending on the initial conditions. In particular, single pulse seeds yielded $\sim 10$ ps stable single pulse solutions, but if the seed was a double pulse with separation 40-200 ps, the simulations converged to a stable molecule with identical separation. This behaviour is consistent with similar modelling of stretched-pulse lasers, where attraction to the stable state is attributed to cross-phase modulation and nonlinear gain [27]. We note, however, that for other regimes, separations different from the seed have also been reported [28]. We also remark that when noisy initial conditions were used in this regime, simulations converge to either single or double pulses in a random way depending on the particular noise on the seed.

The blue region corresponds to only stable molecules being observed irrespective of initial conditions (i.e. even for single pulse seeds). Note that the boundaries between the regions in Fig. 2 depend weakly on the separation used for the doublepulse initial conditions. For purposes of reproducibility, we plot

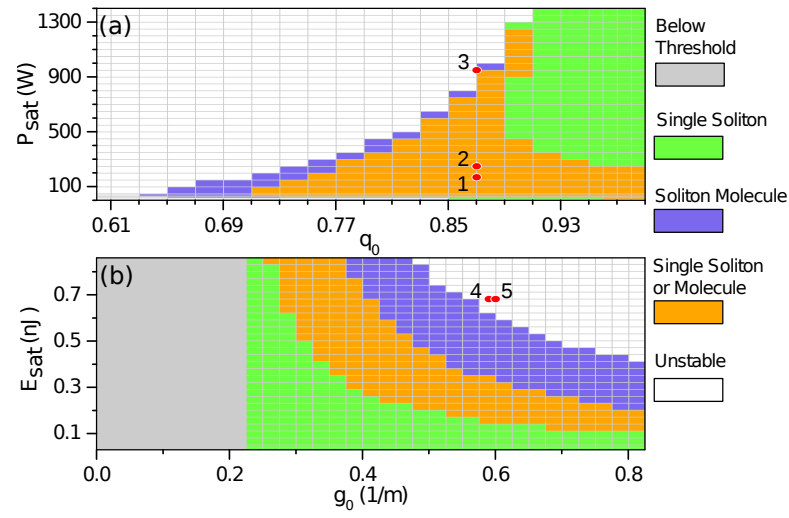

Fig. 2. Stability regimes as a function of: (a) saturable absorber parameters $\left(q_{0}, P_{\text {sat }}\right)$ at fixed $E_{\text {sat }}=0.4 \mathrm{~nJ}, g_{0}=0.78 \mathrm{~m}^{-1}$, and (b) gain parameters $\left(g_{0}, E_{\text {sat }}\right)$ for fixed $P_{\text {sat }}=150 \mathrm{~W}, q_{0}=0.70$. The colored legend describes the different regimes (see text). In (a) the red points $(1,2)$ correspond to the soliton molecule results in Fig. 3. The red points $(3,4,5)$ in (a) and (b) correspond to the results in Fig. 4.

results here for a separation of 150 ps.

The steady state pulse energy varied over the stability map as follows. For Fig. 2(a): green region (2.0-2.6 nJ ); orange region (2.2-2.9 nJ single pulses, 2.2-3.1 nJ double pulses); blue region (2.3-3.1 nJ). For Fig. 2(b): green region (12 pJ-0.73 nJ ); orange region (0.68-1.7 nJ single pulses, $0.70-1.8 \mathrm{~nJ}$ double pulses); blue region $(1.5-2.9 \mathrm{~nJ})$.

As discussed above, double pulse seeds of separation 40200 ps resulted in stable molecules (orange and blue regions in Fig. 2). However, decreasing the separation below $\sim 40$ ps was found to induce variation in the relative phase between the molecule components, arising from enhanced interactions between the pulse tails [28, 29].

Such phase variations manifest experimentally through changes in the fringe structure of the molecule spectrum with roundtrips [30], previously seen in real time spectral measurements $[2,6,7,15]$. Specifically, for a molecule consisting of two pulses centred on $\omega_{0}$ with temporal separation $T$ and relative phase $\varphi$, the spectrum is: $S(\omega)=\left|\tilde{E}\left(\omega-\omega_{0}\right)\right|^{2}(1+\cos [2 \pi(\omega-$ $\left.\left.\left.\omega_{0}\right) / \Omega_{\mathrm{p}}+\varphi\right]\right)$ where $\tilde{E}\left(\omega-\omega_{0}\right)$ is the spectral amplitude of each pulse, and $\Omega_{p}=2 \pi / T$ gives the frequency separation between adjacent fringes in the spectrum. As $\varphi$ varies with roundtrip, the fringe structure is then displaced.

Our model reproduces this behaviour, and Fig. 3 shows two examples. Fig. 3(a-d) corresponds to Point 1 in Fig. 2(a) with $P_{\text {sat }}=170 \mathrm{~W}, q_{0}=0.87$. Here Fig. 3(a) shows the time-domain profile of the molecule at the EDF output after a particular roundtrip, and by following the spectrum over 500 subsequent roundtrips (zooming into a $10 \mathrm{~nm}$ span near $1550 \mathrm{~nm}$ ), Fig. 3(b) shows the spectral modulation oscillating with a $\sim 65$ roundtrip period. The relative phase between the molecule components can be extracted from the spectrum [30] or in our case directly from the complex field, and is shown in Fig. 3(c).

This oscillatory phase has been described as analogous to molecular vibrations [6], attributed to roundtrip-to-roundtrip peak power oscillations experienced by the two components of the molecule [29]. These oscillations are seen in our simulations, and shown in Fig. 3(d) at the EDF output. They arise physically from pulse tail-mediated interaction and the effects 
of gain and loss, including gain saturation [31]. Our simulations confirm quantitatively that these oscillations induce the observed spectral phase variation. Specifically, consider two pulses in a molecule of peak power $P_{1}(z)$ and $P_{2}(z)$ as they circulate at any point in the cavity $z$. Accounting for the different nonlinearity of the cavity segments by a $z$-dependent $\gamma(z)$, the difference in nonlinear phase between the component pulses $\delta \phi_{k+1}$ after roundtrip $k+1$ is related to that after roundtrip $k$ by $\delta \phi_{k+1}=\delta \phi_{k}+\int \gamma(z)\left[P_{2}(z)-P_{1}(z)\right] d z$, where the integral is performed around the cavity (over the $k$-th roundtrip). From the simulations which yield peak power at every point in the cavity, it is straightforward to calculate this nonlinear phase and compare with the phase computed from the full field. For roundtrips above 250, Fig. 3(c) compares the phase variation calculated from the peak power integration (dashed black line) with that from the complex field (solid green line) and there is essentially exact agreement.

The results in Fig. 3(e-h) correspond to Point 2 in Fig. 2(a) with $P_{\text {sat }}=250 \mathrm{~W}, q_{0}=0.87$. At this higher saturation power, the relative phase between the molecule components shows linear displacement with roundtrip (sometimes referred as an infinite or diverging phase [6]) with periodic oscillation about this linear trend (see inset in Fig. 3(g)). Again computing the nonlinear phase variation from the peak power variation yields essentially exact agreement with that obtained directly from the simulation complex field.

These simulations yield insight into the conditions under which the different behaviour in Fig. 3 is seen. Specifically, if the peak powers of the constituent pulses vary about a common mean as in Fig 3(d), then the average position of the fringes remains constant with roundtrip but the fringes display periodic oscillation. In contrast, if the peak powers of the pulses vary about distinct means as in Fig 3(h) then the fringes show an overall linear displacement with roundtrip and also display oscillation about this linear trend.

Outside regimes of stability or internal motion, the white regions in Fig. 2 correspond to simulations where no convergence was seen, and large temporal, spectral and energy fluctuations over multiple roundtrips. The evolution here depended sensitively on initial conditions, with different random noise yielding very different behaviour. Figure 4 shows typical results, using initial conditions of 6 ps pulses with uniformly-distributed $100 \%$ multiplicative noise. These conditions were found to reproduce transitions between qualitatively different behaviour over 100's of roundtrips as seen in experiments.

Figure 4(a) corresponds to Point 3 in Fig. 2(a) with $P_{\text {sat }}=$ $950 \mathrm{~W}, q_{0}=0.87$. Figures. 4 (b) and (c) correspond to Points 4 and 5 in Fig. 2(b) with $E_{\text {sat }}=0.68 \mathrm{~nJ}, g_{0}=0.59 \mathrm{~m}^{-1}$ and $E_{\text {sat }}=$ $0.68 \mathrm{~nJ}, g_{0}=0.60 \mathrm{~m}^{-1}$ respectively. For all cases, we plot (top to bottom): energy fluctuations; spectral evolution; temporal intensity evolution; temporal autocorrelation function evolution. Although simulations yield the temporal intensity directly, we also plot the autocorrelation because it is this that is usually derived from experimental measurements [30]. These results are remarkable in yielding features of spectral evolution similar to those seen in recent experiments. Figure 4(a) for example shows intermittent molecule states (similar to experiments in Ref. [2]), Fig. 4(b) shows metastable molecule states interrupted by explosion dynamics (similar to experiments in Refs. [15, 32]) and Fig. 4(c) shows the decay of a molecule state into essentially a broadband chaotic field with intermittent very short-lived single pulse states (similar to experiments in Ref. [15] ).

The conclusions are as follows. Firstly, a scalar model for a
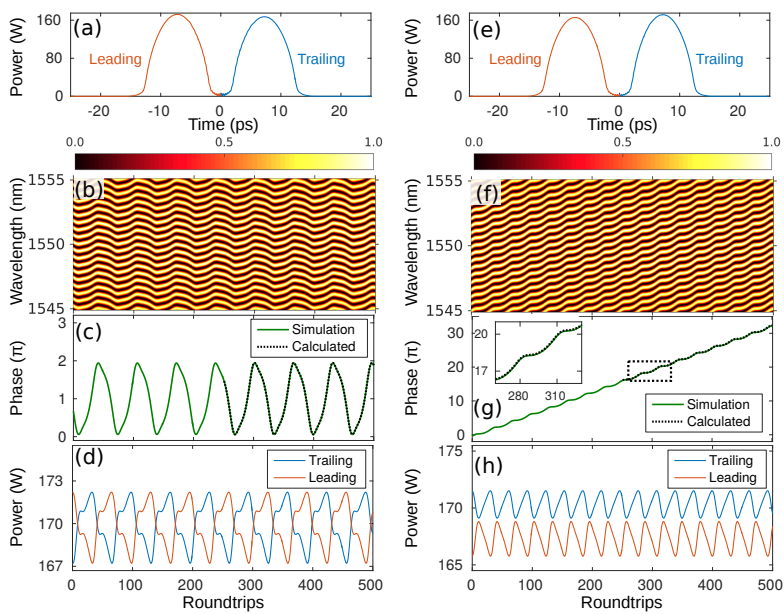

Fig. 3. Molecule internal motion. Results in (a-d) correspond to point 1 in Fig. 2(a) and show oscillating phase. We plot: (a) temporal intensity showing trailing (blue) and leading (red) pulses at one particular roundtrip. Following 500 roundtrips we show: (b) spectral evolution over a $10 \mathrm{~nm}$ span showing fringe oscillations; (c) corresponding phase evolution computed from the simulated field (green), and calculated only from the peak power (black dashed, shown from roundtrips 250-500); (d) peak power evolution for leading (red) and trailing (blue) pulses. Results in (e-h) correspond to point 2 in Fig. 2(a) for a diverging phase. The seed in both cases was a Gaussian double pulse with 14.5 ps separation.

dissipative soliton laser has been shown to reproduce a range of instability behaviour seen in experiments. For soliton molecule evolution, the model reproduces oscillating and diverging phase, and in both cases allows the phase evolution to be associated with variations in peak power of the molecule components. To our knowledge, this is the first time that the link between peak power evolution and oscillatory or divergent phase has been shown quantitatively. Outside the stability regimes, a range of spectral and autocorrelation evolution dynamics were observed similar to recent experiments. It is particularly significant that although the results in Refs $[2,15]$ were obtained using nonlinear polarization evolution as the saturable absorber mechanism, our simulations yield similar results assuming only scalar propagation and generic models for gain and saturable absorption.

This suggests that the instabilities in recent experiments are not contingent on gain dynamics or nonlinear polarization evolution, but rather arise from basic NLSE interactions. Although quantitative comparison with experiment may require fully realistic models, the essential physical features can be reproduced using a simpler reduced approach. In the context of Fig. 4, our results confirm previous studies showing how complex instabilities appear to be intrinsic to the interaction between nonlinearity, dispersion and dissipation [16]. Of course, understanding how particular instabilities are triggered remains an open question, but we anticipate that the ability to study such problems using a scalar model will yield new general insights, also with regards to issues of multistability and higher-order molecule formation.

Funding. Agence Nationale de la Recherche (ANR-15-IDEX0003, ANR-17-EURE-0002); Academy of Finland (298463, 318082 Flagship PREIN).

Disclosures. The authors declare no conflicts of interest. 
(a)
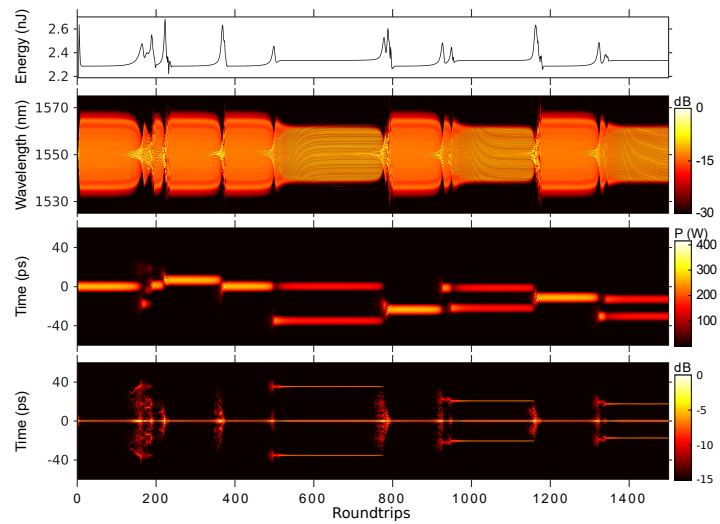

(b)

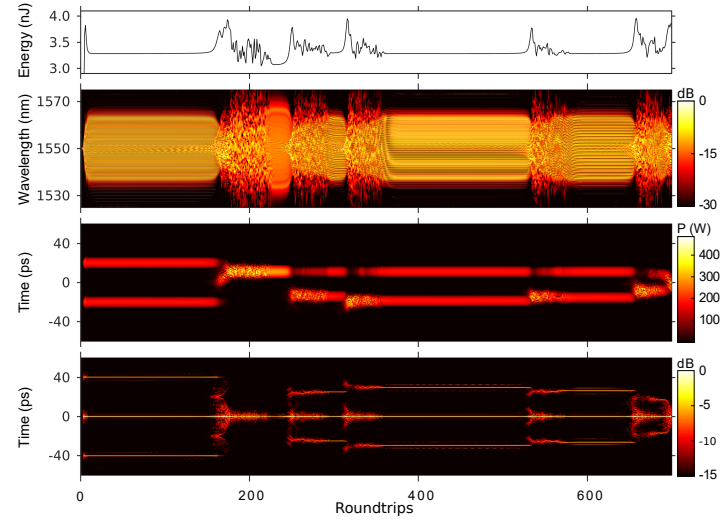

(c)

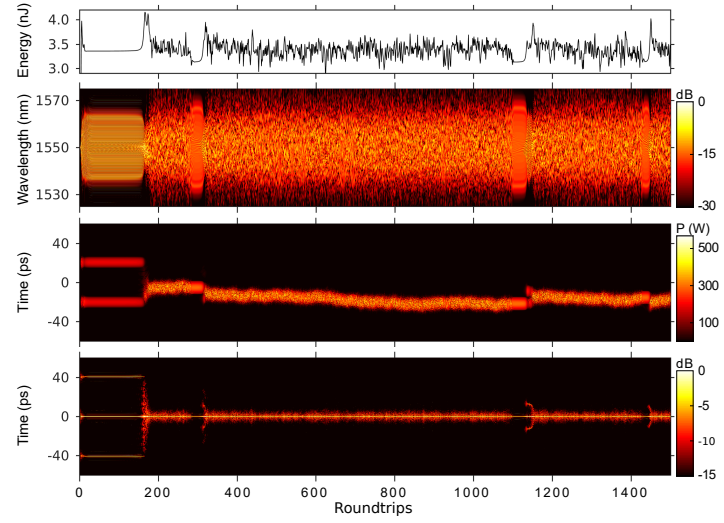

Fig. 4. Unstable regimes showing (top to bottom) energy (nJ), spectrum (normalized in $\mathrm{dB}$ ), instantaneous power $\mathrm{P}(\mathrm{W})$, temporal autocorrelation (normalized in dB). (a) Point 3 in Fig. 2(a) with initial condition of a 6 ps Gaussian pulse with noise; (b) Point 4 in Fig. 2(b) with initial condition of a double pulse with noise (separation 40 ps); (c) Point 5 in Fig. 2(b) with initial condition of a double pulse with noise (separation 40 ps.)

\section{REFERENCES}

1. X. Liu, X. Yao, and Y. Cui, Phys. Rev. Lett. 121, 023905 (2018).

2. J. Peng and H. Zeng, Laser \& Photonics Rev. 12, 1800009 (2018).

3. H.-J. Chen, M. Liu, J. Yao, S. Hu, J.-B. He, A.-P. Luo, W.-C. Xu, and Z.-C. Luo, Opt. Express 26, 2972 (2018).

4. X. Liu and Y. Cui, Adv. Photonics 1, 016003 (2019).

5. X. Liu and M. Pang, Laser \& Photonics Rev. 13, 1800333 (2019).

6. K. Krupa, K. Nithyanandan, U. Andral, P. Tchofo-Dinda, and P. Grelu, Phys. Rev. Lett. 118, 243901 (2017).

7. Wang, Z. Q., Nithyanandan, K., Coillet, A., Tchofo-Dinda, P., and Grelu, Ph., Nat. Commun. 10, 830 (2019).

8. A. F. J. Runge, N. G. R. Broderick, and M. Erkintalo, Optica. 2, 36
(2015).

9. Z. Wang, Z. Wang, Y. Liu, R. He, J. Zhao, G. Wang, and G. Yang, Opt. Lett. 43, 478 (2018).

10. Y. Du, Z. Xu, and X. Shu, Opt. Lett. 43, 3602 (2018).

11. Z.-W. Wei, M. Liu, S.-X. Ming, A.-P. Luo, W.-C. Xu, and Z.-C. Luo, Opt. Lett. 43, 5965 (2018).

12. M. Suzuki, O. Boyraz, H. Asghari, P. Trinh, H. Kuroda, and B. Jalali, Opt. Lett. 43, 1862 (2018).

13. X. Wang, Y.-g. Liu, Z. Wang, Y. Yue, J. He, B. Mao, R. He, and J. Hu, Opt. Express 27, 17729 (2019).

14. J. Peng and H. Zeng, Commun. Phys. 2, 34 (2019).

15. C. Lapre, C. Billet, F. Meng, P. Ryczkowski, T. Sylvestre, C. Finot, G. Genty, and J. M. Dudley, Sci. Reports 9, 1 (2019).

16. N. Akhmediev, J. M. Soto-Crespo, and G. Town, Phys. Rev. E 63 , 056602 (2001).

17. S. K. Turitsyn, B. G. Bale, and M. P. Fedoruk, Phys. Reports 521, 135 (2012).

18. W. Chang, J. M. Soto-Crespo, P. Vouzas, and N. Akhmediev, Phys. Rev. E 92, 022926 (2015).

19. R. He, Z. Wang, Y. Liu, Z. Wang, H. Liang, S. Han, and J. He, Opt. Express 26, 33116 (2018).

20. P. Wang, X. Xiao, P. Grelu, and C. Yang, IEEE Photonics J. 9, 1 (2017).

21. Y. Du and X. Shu, Opt. Express 26, 5564 (2018).

22. S. H. Nazari and A. S. Arabanian, J. Opt. Soc. Am. B 35, 2633 (2018).

23. B. Oktem, C. Ülgüdür, and F. O. Ilday, Nat. Photonics 4, 307 (2010).

24. R. I. Woodward, J. Opt. 20, 033002 (2018).

25. I. A. Yarutkina, O. V. Shtyrina, M. P. Fedoruk, and S. K. Turitsyn, Opt. Express 21, 12942 (2013).

26. W. H. Renninger, A. Chong, and F. W. Wise, IEEE J. Sel. Top. Quantum Electron. 18, 389 (2012).

27. M. Olivier and M. Piché, Opt. Express 17, 405 (2009).

28. X. M. Liu, X. X. Han, and X. K. Yao, Sci. Reports 6, 34414 (2016).

29. J. M. Soto-Crespo and N. N. Akhmediev, J. Opt. Soc. Am. B 16, 674 (1999).

30. Herink, G., Kurtz, F., Jalali, B., Solli, D. R., and Ropers, C., Science 356, 50 (2017).

31. A. Zavyalov, R. Iliew, O. Egorov, and F. Lederer, Phys. Rev. A 80, 043829 (2009).

32. Y. Yu, Z.-C. Luo, J. Kang, and K. K. Y. Wong, Opt. Lett. 43, 4132 (2018). 